\title{
Crossover from a continuum study of chiral susceptibility
}

\author{
Min $\mathrm{He}^{a}$, Fei $\mathrm{Hu}^{a}$, Wei-Min Sun ${ }^{a, b}$, and Hong-Shi Zong ${ }^{a, b}$ \\ a Department of Physics, Nanjing University, Nanjing 210093, P. R. China and \\ b Joint Center for Particle, Nuclear Physics and Cosmology, Nanjing 210093, China
}

\begin{abstract}
We derive a model-independent integral formula for chiral susceptibility and attempt to present a continuum model study of it within the framework of Dyson-Schwinger Equations. An appropriate regularization is implemented to remove the temperature-independent quadratic divergence inherent in this quantity. While it demonstrates a second-order phase transition characteristic in the chiral limit, the result obtained supports a crossover at physical current quark masses, which is in good agreement with recent lattice studies.
\end{abstract}

Key-words: chiral susceptibility, QCD thermal transition, crossover

E-mail: zonghs@chenwang.nju.edu.cn.

PACS Numbers: 11.10.Wx, 11.15.Tk, 11.30.Qc 


\section{INTRODUCTION}

Quantum Chromodynamics (QCD) underlies our current understanding of strong interaction and explains why and how the fundamental constituent, namely quarks and gluons are bound into nucleons, pions, etc. The QCD vacuum is rather complex and intriguing: while it features the overwhelming spontaneous chiral symmetry breaking and color confinement, it is believed to be heated and undergo a transition at high enough temperature [1, 2, 3, 4]. As a result, quarks and gluons are no longer confined inside hadrons and a new state of strongly interacting matter, the so-called quark-gluon plasma (QGP) is expected to form. Meanwhile, the chiral symmetry gets restored. It is a goal to detect such a new state of matter for the on-going heavy-ion collision experiments at the BNL Relativistic Heavy-Ion Collider (RHIC) and the near future Large Hadron Collider (LHC). It is widely speculated that this QCD-related transition may also be of direct relevance for the evolution of the early Universe $[1,[5]$

Although lattice QCD has established the existence of such a thermal transition, the nature of this transition (its order, or, whether is is a real phase transition) has been a long time debate [1]. It is not until recently that Y. Aoki and his collaborators, by exploiting quite demanding and powerful lattice simulations, assert unambiguously that QCD evolves smoothly with temperature; that is, there is no thermodynamic phase transition for three flavors (u, d, s-quark) with physical current masses, but instead a smooth crossover [2, 6]. Such a conclusion rules out some scenarios and speculations over the evolution of the early Universe [1]. In fact, the nature of the QCD thermal transition would lay an imprint on the Universe's evolution and thus affect our understanding of the latter. For example, the previously prevailing strong first-order transition scenario predicts inhomogeneous nucleosynthesis and later formation of cold dark matter clumps [5, 7]. Nowadays, people tend to believe that the nature of QCD thermal transition depends on the quark content and current quark masses [8, 9]. In the chiral limit, the chiral phase transition is likely to be of second order for two flavors and first order for three flavors. For infinite or very large current quark masses, the transition turns out to be a first-order deconfinement transition. For intermediate current masses, one may end up with a crossover, instead of a real phase transition, meaning that thermodynamic quantities would show a rapid change, as opposed to a jump, in a narrow temperature range. 
As is well known, first principle lattice simulations provide the prior choice to address the thermal QCD transition problem, because of its intrinsic nonperturbative nature. In this regard, recently Y. Aoki and collaborators [6] achieved a large scale computing and identified the nature of three-flavor QCD finite temperature transition as a smooth crossover at physical quark masses. On the other hand, other than lattice simulations, continuum nonperturbative model study can be a complementary approach to thermal QCD transition.

Following Y. Aoki et al. in Refs. [2, 6], we present a continuum model study of the interesting chiral susceptibility in the present work, from whose behavior as temperature varies we identify the nature of two-flavor QCD thermal transition in the case of physical current quark masses as well as in the chiral limit.

We first derive a model-independent analytical formula, which expresses chiral susceptibility as a integral in terms of dressed propagators and dressed scalar vertex. The quadratic divergence inherent in this susceptibility is then demonstrated and an appropriate renormalization procedure is specified. In the section that follows, this result is then illustrated by a model calculation based on an effective interaction in the framework of rainbow DysonSchwinger equation (DSE) and ladder Bethe-Salpeter equation (BSE) approach. The notorious quadratic ultraviolet divergence is appropriately subtracted by a delicate numerical procedure. The results obtained are carefully analyzed and a crossover and a second-order phase transition are respectively identified for the cases of physical and zero current quark masses. The final section is devoted to the conclusion and discussion. Throughout this paper, we work with Euclidean space metric: $\left\{\gamma_{\mu}, \gamma_{\nu}\right\}=2 \delta_{\mu \nu}$.

\section{DERIVATION AND RENORMALIZATION}

\section{A. Derivation of an integral formula for chiral susceptibility}

Chiral susceptibility has interested quite a few lattice QCD practitioners and attracted much investigation [3, 6 , 8, 9, 10, 11, 12, 13, 14, 15, 16, 17, 20]. It is defined as

$$
\chi=\frac{T}{V} \frac{\partial^{2}}{\partial m^{2}} \log Z=(-) \frac{\partial}{\partial m}<\bar{\psi} \psi>
$$


where $Z$ denotes the QCD partition function at finite temperature and vanishing chemical potential.

$$
Z=\int \mathcal{D} \bar{\psi} \mathcal{D} \psi \mathcal{D} A \exp \left\{-\int_{0}^{\beta} d \tau \int d^{3} x\left[\bar{\psi}_{f}\left(\gamma \cdot \partial+m_{f}+i g \frac{\lambda^{a}}{2} \gamma \cdot A^{a}\right) \psi_{f}+\frac{1}{4} F_{\mu \nu}^{a} F_{\mu \nu}^{a}\right]\right\}
$$

Here, $\bar{\psi}, \psi$ and $A_{\mu}^{a}$ are subject to anti-periodic and periodic conditions, respectively; and we leave the gauge fixing term and the ghost field term to be understood. Note that chiral susceptibility is defined for two light flavors and hence $m$ in Eq. (1) is supposed to be the degenerate current mass of $\mathrm{u}, \mathrm{d}$ quarks. The second line of that equation also indicates that the chiral susceptibility measures the response of chiral condensate (the order parameter) to a small perturbation of the parameter responsible for explicit breaking of chiral symmetry (the current quark mass).

Formally, using quark propagator we can always write the quark condensate as

$$
<\bar{\psi} \psi>=(-) N_{c} N_{f} \int \frac{d^{4} p}{(2 \pi)^{4}} \operatorname{tr}_{\gamma} G(p, m)
$$

where $t r_{\gamma}$ denotes trace over Dirac indices of the quark propagator, $N_{c}=3$ is the color factor and $N_{f}=2$ denotes two degenerate light flavors. Substituting Eq. (3) into Eq. (1) and adopting the identity

$$
\frac{\partial G(p, m)}{\partial m}=-G(p, m) \frac{\partial G^{-1}(p, m)}{\partial m} G(p, m)
$$

we have

$$
\chi=(-) N_{c} N_{f} \int \frac{d^{4} p}{(2 \pi)^{4}} \operatorname{tr}_{\gamma}\left[G(p, m) \frac{\partial G^{-1}(p, m)}{\partial m} G(p, m)\right] .
$$

We consider the mass $m$ as a constant background scalar field coupled to the quark fields by the term $m \bar{\psi} \psi$. Then $G(p, m)$ is the dressed quark propagator in the presence of such a background field and the derivative of its inverse with respect to the current quark mass yields the so-called one-particle-irreducible (1PI) dressed scalar vertex (a three-point correlation function)

$$
\frac{\partial G^{-1}(p, m)}{\partial m}=\Gamma(p, 0 ; m)
$$

where $p$ is the relative momentum. This relation may be called "scalar Ward identity". Note that the total momentum of the dressed scalar vertex vanishes because the background scalar field $m$ is a constant that takes no momentum. Substituting Eq. (6) into Eq. (5) gives

$$
\chi=(-) N_{c} N_{f} \int \frac{d^{4} p}{(2 \pi)^{4}} \operatorname{tr}_{\gamma}[G(p, m) \Gamma(p, 0 ; m) G(p, m)]
$$


This is the integral formula for chiral susceptibility at zero temperature. Diagrammatically, the right-hand-side of Eq. (7) expresses the Feynman diagram for the color-singlet scalar vacuum polarization at zero total momentum. This clearly shows that chiral susceptibility at zero temperature equals the color-singlet scalar vacuum polarization at zero total momentum.

According to finite-temperature field theory (see, e.g., [18]), the corresponding finite temperature version is obtained by replacing the integration over the fourth component of momentum with summation over fermion Matsubara frequencies:

$$
\chi(T)=(-) N_{c} N_{f} T \sum_{n=-\infty}^{+\infty} \int \frac{d^{3} p}{(2 \pi)^{3}} \operatorname{tr}_{\gamma}\left[G\left(p_{n}, m\right) \Gamma\left(p_{n}, 0 ; m\right) G\left(p_{n}, m\right)\right]
$$

where $p_{n}=\left(\vec{p}, \omega_{n}\right)$ with Matsubara frequencies $\omega_{n}=(2 n+1) \pi T$. Here it should be noted that in the chiral limit the above expression for the chiral susceptibility reduces to the corresponding expression (Eq. (7)) in Ref. [19].

So we have derived a model-independent integral formula which expresses the chiral susceptibility in terms of the dressed quark propagator and the dressed scalar vertex, both of the latter being basic quantities in quantum field theory (QFT). The DSE-BSE approach provides a desirable framework to calculate these quantities non-perturbatively and hence the chiral susceptibility.

\section{B. Quadratic divergence and renormalization}

There resides an additive quadratic ultraviolet divergence in the chiral susceptibility, which can manifest itself even in the chiral limit [2, 6]. To see this unambiguously, we calculate the chiral susceptibility in the case of free quark gas, because all quantities tend to the free case counterparts in the ultraviolet limit (asymptotic freedom) and all divergences originate from the ultraviolet limit. In this case, the dressed scalar vertex reduces to the bare one, i.e.

$$
\Gamma\left(p_{n}, 0\right) \rightarrow \mathbf{1}
$$

and the free quark propagator in the chiral limit reads

$$
G^{\text {free }}\left(p_{n}\right)=\frac{1}{i \gamma \cdot p_{n}}
$$


Substituting Eqs. (9) and (10) into Eq. (8), one gets

$$
\chi^{\text {free }}(T)=4 N_{c} N_{f} T \sum_{n=-\infty}^{+\infty} \int \frac{d^{3} p}{(2 \pi)^{3}} \frac{1}{\omega_{n}^{2}+\vec{p}^{2}} .
$$

With the aid of the identity

$$
T \sum_{n=-\infty}^{+\infty} \frac{x}{\omega_{n}^{2}+x^{2}}=\frac{1}{2}\left(1-2 n_{F}(x)\right)
$$

where $n_{F}(x)=\frac{1}{e^{x / T}+1}$ is the Fermi-Dirac statistics function, we have

$$
\chi^{\text {free }}(T)=2 N_{c} N_{f} \int \frac{d^{3} p}{(2 \pi)^{3}} \frac{1}{|\vec{p}|}\left(1-2 n_{F}(|\vec{p}|)\right) .
$$

The second part of the integrand can be integrated out and gives a purely temperaturedependent finite result: $-\frac{N_{c} N_{f}}{6} T^{2}$. On the contrary, the first part yields an additive quadratic divergence. This is in striking contrast to the free quark-number susceptibility, which is a definitely finite quantity proportional to $T^{2}$ and therefore vanishes at zero temperature [19].

Actually, there always contains an inherent additive quadratic divergence in chiral susceptibility. One dose not really have to address the issue of the regularization of this quadratic divergence, when one works with a hard cut-off in the numerical integration (e.g., lattice simulations), and is concerned only with the temperature-dependent behavior of the susceptibility, not so much on its absolute value [21]. However, a correct renormalization procedure is important and indispensable if one wants to get correct physics in the continuum limit [2]. In Ref. [19], we identified the disconnected part of the chiral susceptibility and argued that it is of major relevance and free from quadratic ultraviolet divergence. However, we were unable to derive the disconnected chiral susceptibility directly from a first-principle approach such as the functional integral method. Looked at from this point, the identification of the disconnected chiral susceptibility is not yet absolutely "waterproof" and solid enough. So in this paper we follow the renormalization procedure in Ref. [2], which is to subtract the zero temperature susceptibility and study the difference between $T \neq 0$ and $T=0$. That is to say, we define the renormalized chiral susceptibility by

$$
\chi_{R}(T)=\chi(T)-\chi
$$

where $\chi(T)$ and $\chi$ are provided by Eq. (8) and Eq. (7), respectively. The justification for this renormalization is two-fold: firstly, the quadratic divergence is additive and temperature- 
independent and hence can be really removed by subtracting the zero-temperature susceptibility; secondly, this subtraction will never affect the genuine temperature effects of chiral susceptibility that we are interested in.

Having specified the renormalization procedure, in the next section, we will focus on the model numerical calculation of the renormalized chiral susceptibility $\chi_{R}(T)$ within the framework of DSE-BSE.

\section{MODEL NUMERICAL CALCULATION}

Dyson-Schwinger equations provide a non-perturbative, continuum approach for the exploration of strong interaction physics [21]. Derived from QCD's Euclidean space generating functional, they constitute a tower of enumerable coupled integral equations whose solutions are the n-point Green's functions. A consistent truncation scheme that makes the DSE calculations tractable is the so-called rainbow-ladder approximation. Such a rainbow-DSE and ladder-BSE approach has been used extensively in the investigation of spontaneously chiral symmetry breaking and confinement [22] and also found successful applications in calculating light pseudo-scalar and vector meson observables [23, 24] as well as in describing strong interaction at finite temperature and/or density [25]. The subsequent model calculation of chiral susceptibility will also be conducted systematically and consistently in the rainbow-DSE and ladder-BSE framework with an effective gluon propagator.

\section{A. The zero-temperature chiral susceptibility}

We first calculate the zero-temperature chiral susceptibility with Eq. (7). This involves the calculation of dressed quark propagator and dressed scalar vertex. The former is solved from the rainbow gap equation

$$
G^{-1}(p, m)=i \gamma \cdot p+m+\frac{4}{3} \int \frac{d^{4} q}{(2 \pi)^{4}} g^{2} D_{\mu \nu}^{e f f}(p-q) \gamma_{\mu} G(q, m) \gamma_{\nu}
$$

and the latter satisfies the ladder inhomogeneous BSE (the total momentum of the dressed scalar vertex has been set equal to zero)

$$
\Gamma(p, 0 ; m)=\mathbf{1}-\frac{4}{3} \int \frac{d^{4} q}{(2 \pi)^{4}} g^{2} D_{\mu \nu}^{e f f}(p-q) \gamma_{\mu} G(q, m) \Gamma(q, 0 ; m) G(q, m) \gamma_{\nu},
$$


where $D_{\mu \nu}^{e f f}(p-q)$ is the effective gluon propagator, which is usually a phenomenological input in practice (it should also be noted that the analytical structure of the gluon propagator has been explored from numerical solutions of coupled DSEs of quarks, gluons and ghosts and from fits to lattice data in recent literatures, see Ref. [26] and references therein).

At the moment, we pause for a while and give a brief proof that the rainbow-ladder truncation scheme of DSE-BSE respects the scalar Ward identity (Eq. (6)). Taking the derivative with respect to $m$ on both sides of Eq. (15) and using again the identity Eq. (4), one arrives at

$$
\frac{\partial G^{-1}(p, m)}{\partial m}=\mathbf{1}-\frac{4}{3} \int \frac{d^{4} q}{(2 \pi)^{4}} g^{2} D_{\mu \nu}^{e f f}(p-q) \gamma_{\mu} G(q, m) \frac{\partial G^{-1}(q, m)}{\partial m} G(q, m) \gamma_{\nu}
$$

Comparing Eq. (16) and Eq. (17), one sees that $\Gamma(p, 0 ; m)$ and $\partial G^{-1}(p, m) / \partial m$ satisfy the same integral equation and therefore they must be equal, i.e., the scalar Ward identity holds at the level of the rainbow-ladder truncation. That is to say, the rainbow-ladder DSEBSE model calculation respects the scalar Ward identity, whatever form the effective model gluon propagator takes. In fact, the rainbow-ladder truncation scheme of DSE-BSE itself is consistent in a broader sense. In addition to the scalar Ward identity, this truncation scheme preserves the Abelian vector Ward-Takahashi identity and the axial-vector Ward-Takahashi identity [23]. It is the preservation of the latter identity that guarantees an understanding of chiral symmetry and its dynamical breaking in the rainbow-ladder DSE-BSE framework without a fine-tuning of model-dependent parameters [23].

In this paper, we will employ the rank-2 confining separable model gluon propagator

$$
g^{2} D_{\mu \nu}^{e f f}(p-q) \rightarrow \delta_{\mu \nu} D\left(p^{2}, q^{2}, p \cdot q\right)=\delta_{\mu \nu}\left[D_{0} f_{0}\left(p^{2}\right) f_{0}\left(q^{2}\right)+D_{1} f_{1}\left(p^{2}\right) p \cdot q f_{1}\left(q^{2}\right)\right]
$$

with $f_{i}\left(p^{2}\right)=\exp \left(-p^{2} / \Lambda_{i}^{2}\right), i=1,2$. It is found to be very successful in describing light flavor (including $\mathrm{u}, \mathrm{d}$, s-quarks) pseudo-scalar and vector mesons with parameters $\Lambda_{0}=0.638 \mathrm{GeV}$, $\Lambda_{1} / \Lambda_{0}=1.21, D_{0} \Lambda_{0}^{2}=260.0, D_{1} \Lambda_{1}^{4}=130.0$ and $m=0.0053 \mathrm{GeV}$ [27, 28].

To proceed our model calculation, note that the quark propagator can be decomposed in terms of two independent Lorentz structures (no $\gamma_{5}$-related structure appears in pure strong interaction)

$$
G^{-1}(p, m)=i \gamma \cdot p A\left(p^{2}, m\right)+B\left(p^{2}, m\right)
$$

Upon substituting Eqs. (18) and (19) into Eq. (15), the latter is converted into two coupled 
integral equations $\left(s=p^{2}\right)$

$$
\begin{aligned}
a(m) & =\frac{D_{1}}{24 \pi^{2}} \int_{0}^{\infty} d s \frac{s^{2} f_{1}(s)\left[1+a(m) f_{1}(s)\right]}{s\left[1+a(m) f_{1}(s)\right]^{2}+\left[m+b(m) f_{0}(s)\right]^{2}} \\
b(m) & =\frac{D_{0}}{3 \pi^{2}} \int_{0}^{\infty} d s \frac{s f_{0}(s)\left[m+b(m) f_{0}(s)\right]}{s\left[1+a(m) f_{1}(s)\right]^{2}+\left[m+b(m) f_{0}(s)\right]^{2}}
\end{aligned}
$$

with $A\left(p^{2}, m\right)=1+a(m) f_{1}\left(p^{2}\right)$ and $B\left(p^{2}, m\right)=m+b(m) f_{0}\left(p^{2}\right)$.

As for the dressed scalar vertex, its general form from Lorentz structure analysis reads

$$
\Gamma(p, 0 ; m)=F\left(p^{2}, m\right) \cdot \mathbf{1}+i \gamma \cdot p G\left(p^{2}, m\right)
$$

but only the first term provides the leading behavior [29]. We shall keep only this term for simplicity. Putting this ansatz $\Gamma(p, 0 ; m)=F\left(p^{2}, m\right) \cdot \mathbf{1}$, the model gluon propagator Eq. (18) and the quark propagator Eq. (19) into Eq. (16), one has after completing some algebras

$$
\Gamma(p, 0 ; m)=F\left(p^{2}, m\right)=1+\alpha(m) f_{0}\left(p^{2}\right)
$$

where $\alpha(m)$ satisfies the following equation

$$
\alpha(m)=\frac{16 D_{0}}{3} \int \frac{d^{4} q}{(2 \pi)^{4}} f_{0}\left(q^{2}\right) \times\left[1+\alpha(m) f_{0}\left(q^{2}\right)\right] \times \frac{q^{2} A^{2}\left(q^{2}, m\right)-B^{2}\left(q^{2}, m\right)}{\left[q^{2} A^{2}\left(q^{2}, m\right)+B^{2}\left(q^{2}, m\right)\right]^{2}} .
$$

We point out that the Gaussian factors $f_{0}\left(p^{2}\right)$ and $f_{1}\left(p^{2}\right)$ in the model gluon propagator provide sufficient ultraviolet suppression and justify an effective cut-off for momentum integration in Eqs. $(20,21,24)$. Therefore no multiplicative renormalization is needed.

With the dressed quark propagator $\left(A\left(p^{2}, m\right)\right.$ and $\left.B\left(p^{2}, m\right)\right)$ and then the dressed scalar vertex $(\alpha(m))$ solved, the zero-temperature chiral susceptibility (Eq. (7)) can now be written as

$$
\chi(m)=4 N_{c} N_{f} \int \frac{d^{4} p}{\left(2 \pi^{4}\right)}\left[1+\alpha(m) f_{0}\left(p^{2}\right)\right] \times \frac{p^{2} A^{2}\left(p^{2}, m\right)-B^{2}\left(p^{2}, m\right)}{\left[p^{2} A^{2}\left(p^{2}, m\right)+B^{2}\left(p^{2}, m\right)\right]^{2}}
$$

and calculated out. Of course, $\chi(m)$ takes an additive quadratic divergence, which serves to cancel that one contained in the temperature-dependent chiral susceptibility.

\section{B. The finite-temperature chiral susceptibility}

In recent years, the rainbow-ladder truncation DSE models are extended to describe the finite temperature properties of QCD and its chiral phase transition [25]. As for the foregoing confining separable model, the extension to finite temperature is systematically 
accomplished by transcription of the Euclidean quark four-momentum via $q \rightarrow q_{n}=\left(\vec{q}, \omega_{n}\right)$, where $\omega_{n}=(2 n+1) \pi T$ are the fermion Matsubara frequencies, and no new parameters are introduced [28]. This means that the effective gluon propagator at finite temperature is modeled as

$$
g^{2} D_{\mu \nu}^{e f f}\left(p_{k}-q_{n}\right)=\delta_{\mu \nu}\left[D_{0} f_{0}\left(p_{k}^{2}\right) f_{0}\left(q_{n}^{2}\right)+D_{1} f_{1}\left(p_{k}^{2}\right) p_{k} \cdot q_{n} f_{1}\left(q_{n}^{2}\right)\right]
$$

with $p_{k}^{2}=\vec{p}^{2}+\omega_{k}^{2}$. The subsequent calculations involving temperature-dependent dressed quark propagator and scalar vertex will be parallel to those of the zero-temperature case specified in the last subsection.

Firstly, the finite temperature version of the rainbow-DSE for the dressed quark propagator Eq. (15) reads

$$
G^{-1}\left(p_{k}, m\right)=i \gamma \cdot p_{k}+\frac{4}{3} T \sum_{n=-\infty}^{+\infty} \int \frac{d^{3} p}{(2 \pi)^{3}} g^{2} D_{\mu \nu}^{e f f}\left(p_{k}-q_{n}\right) \gamma_{\mu} G\left(q_{n}, m\right) \gamma_{\nu}
$$

Its solution now consists of three independent amplitudes due to the breaking of $O(4)$ symmetry down to $O(3)$ symmetry [25]:

$$
G^{-1}\left(p_{n}, m\right)=i \vec{\gamma} \cdot \vec{p} A\left(p_{n}^{2}, m\right)+i \gamma_{4} \omega_{n} C\left(p_{n}^{2}, m\right)+B\left(p_{n}^{2}, m\right)
$$

Substituting Eq. (28) into Eq. (27), one easily obtains solutions of the form $A\left(p_{n}^{2}, m\right)=$ $1+a(T, m) f_{1}\left(p_{n}^{2}\right), B\left(p_{n}^{2}, m\right)=m+b(T, m) f_{0}\left(p_{n}^{2}\right)$, and $C\left(p_{n}^{2}, m\right)=1+c(T, m) f_{1}\left(p_{n}^{2}, m\right)$, where $a(T, m), b(T, m)$ and $c(T, m)$ are functions of $T$ satisfying the following three coupled nonlinear equations:

$$
\begin{aligned}
& a(T, m)=\frac{8 D_{1}}{9} T \sum_{n=-\infty}^{+\infty} \int \frac{d^{3} p}{(2 \pi)^{3}} f_{1}\left(p_{n}^{2}\right) \vec{p}^{2}\left[1+a(T, m) f_{1}\left(p_{n}^{2}\right)\right] d^{-1}\left(p_{n}^{2}, m\right) \\
& c(T, m)=\frac{8 D_{1}}{3} T \sum_{n=-\infty}^{+\infty} \int \frac{d^{3} p}{(2 \pi)^{3}} f_{1}\left(p_{n}^{2}\right) \omega_{n}^{2}\left[1+c(T, m) f_{1}\left(p_{n}^{2}\right)\right] d^{-1}\left(p_{n}^{2}, m\right) \\
& b(T, m)=\frac{16 D_{0}}{3} T \sum_{n=-\infty}^{+\infty} \int \frac{d^{3} p}{(2 \pi)^{3}} f_{0}\left(p_{n}^{2}\right)\left[m+b(T, m) f_{0}\left(p_{n}^{2}\right)\right] d^{-1}\left(p_{n}^{2}, m\right),
\end{aligned}
$$

where $d\left(p_{n}^{2}, m\right)=\vec{p}^{2} A^{2}\left(p_{n}^{2}, m\right)+\omega_{n}^{2} C^{2}\left(p_{n}^{2}, m\right)+B^{2}\left(p_{n}^{2}, m\right)$. These equations are numerically solved. Note that the confining separable model quite facilitates summation over frequencies as well as the three-dimensional integration owing to the sufficient ultraviolet suppression guaranteed by the Gaussian functions $f_{0}\left(p_{n}^{2}\right)$ and $f_{1}\left(p_{n}^{2}\right)$. As a result, no renormalization is needed, which is the same as the zero-temperature case. 
We now turn to the dressed scalar vertex. The finite temperature version of the inhomogeneous ladder BSE for the dressed scalar vertex (Eq. (16)) with vanishing total momentum reads

$$
\Gamma\left(p_{k}, 0 ; m\right)=\mathbf{1}-\frac{4}{3} T \sum_{n=-\infty}^{+\infty} \int \frac{d^{3} p}{(2 \pi)^{3}} g^{2} D_{\mu \nu}^{e f f}\left(p_{k}-q_{n}\right) \gamma_{\mu} G\left(q_{n}, m\right) \Gamma\left(q_{n}, 0 ; m\right) G\left(q_{n}, m\right) \gamma_{\nu}
$$

The solution of Eq. (32) takes the general form

$$
\Gamma\left(p_{n}, 0, m\right)=F\left(p_{n}^{2}, m\right) \cdot \mathbf{1}+i \vec{\gamma} \cdot \vec{p} G_{l}\left(p_{n}^{2}, m\right)+i \gamma_{4} \omega_{n} G_{t}\left(p_{n}^{2}, m\right)
$$

The structure $F\left(p_{n}^{2}, m\right)$ makes the leading-order contribution [30]. In order to be consistent with the zero-temperature case and for simplicity as well, we shall keep only this term and expect it would represent well the temperature-dependent behavior of the dressed scalar vertex. Putting this ansatz $\Gamma\left(p_{n}, 0 ; m\right)=F\left(p_{n}^{2}, m\right)$, the model gluon propagator (Eq. (26)) and the quark propagator (Eq. (28)) into Eq. (32), we find

$$
\Gamma\left(p_{n}, 0 ; m\right)=F\left(p_{n}^{2} ; m\right)=1+\alpha(T, m) f_{0}\left(p_{n}^{2}\right)
$$

where $\alpha(T, m)$ satisfies the following equation

$\alpha(T, m)=\frac{16 D_{0}}{3} T \sum_{n=-\infty}^{+\infty} \int \frac{d^{3} q}{(2 \pi)^{3}} f_{0}\left(q_{n}^{2}\right) \times\left[1+\alpha(T, m) f_{0}\left(p_{n}^{2}\right)\right] \times \frac{\vec{q}^{2} A^{2}\left(q_{n}^{2}, m\right)+\omega_{n}^{2} C^{2}\left(q_{n}^{2}, m\right)-B^{2}\left(q_{n}^{2}, m\right)}{\left[\vec{q}^{2} A^{2}\left(q_{n}^{2}, m\right)+\omega_{n}^{2} C^{2}\left(q_{n}^{2}, m\right)+B^{2}\left(q_{n}^{2}, m\right)\right]^{2}}$

Having obtained the amplitudes $A\left(q_{n}^{2}, m\right), B\left(q_{n}^{2}, m\right)$ and $C\left(q_{n}^{2}, m\right)$ from Eqs. (29-31)), we can numerically solve $\alpha(T, m)$. The Gaussian function $f_{0}\left(p_{n}^{2}\right)$ appearing in the numerator of the integrand justifies an effective cutoff for summation over Matsubara frequencies as well as 3-D integration.

Now, with the dressed quark propagator and dressed scalar vertex at finite temperature solved, the finite-temperature chiral susceptibility Eq. (8) can be finally expressed as

$$
\chi(T, m)=4 N_{c} N_{f} T \sum_{n=-\infty}^{+\infty} \int \frac{d^{3} p}{(2 \pi)^{3}}\left[1+\alpha(T, m) f_{0}\left(p_{n}^{2}\right)\right] \frac{\vec{p}^{2} A^{2}\left(p_{n}^{2}, m\right)+\omega_{n}^{2} C^{2}\left(p_{n}^{2}, m\right)-B^{2}\left(p_{n}^{2}, m\right)}{\left[\vec{p}^{2} A^{2}\left(p_{n}^{2}, m\right)+\omega_{n}^{2} C^{2}\left(p_{n}^{2}, m\right)+B^{2}\left(p_{n}^{2}, m\right)\right]^{2}}
$$

The factor "1" (corresponding to the bare part of the dressed scalar vertex) in the square bracket of the integrand would lead to quadratic divergence, which will be removed by subtracting the zero-temperature counterpart in our renormalization treatment. In order for the finite-temperature chiral susceptibility to approach a correct zero-temperature limit and 
then the temperature-independent quadratic divergence to be canceled by the counterpart in the zero-temperature chiral susceptibility, one should employ the same numerical cut-off as

that used in calculating $\chi(m)$ when calculating $\chi(T, m)$, i.e. choose $\left(\vec{p}^{2}+\omega_{n}^{2}\right)_{\max }=\Lambda_{T}^{2} \equiv \Lambda_{0}^{2}$, where $\Lambda_{T}$ and $\Lambda_{0}$ denote the numerical cut-off in calculating $\chi(T, m)$ (Eq. $\left.(36)\right)$ and $\chi(m)$ (Eq. (25)), respectively.

\section{Numerical results for renormalized chiral susceptibility}

Having obtained both the zero-temperature and finite-temperature chiral susceptibility, we are now in a position to calculate the renormalized chiral susceptibility. The numerical results are shown in Fig. 1 and Fig. 2, where $\chi_{R}(T)$ are both scaled by $T^{2}$ and hence dimensionless.

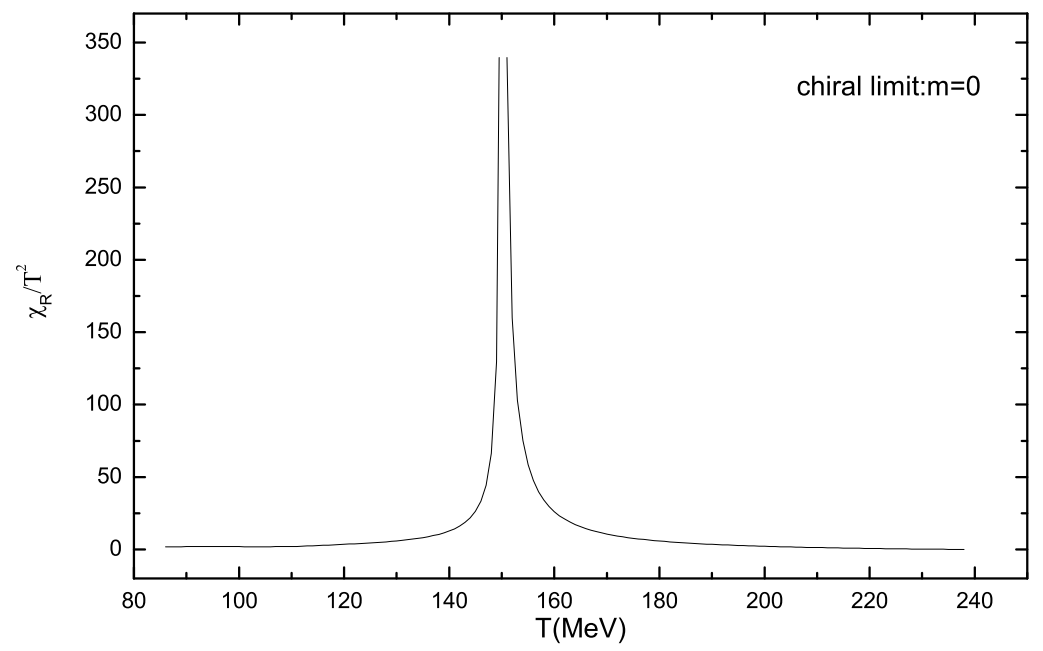

FIG. 1: T-dependence of the renormalized chiral susceptibility in the chiral limit.

From Fig. 1, one sees that in the chiral limit, the renormalized chiral susceptibility exhibits a very narrow, pronounced and in fact, divergent peak at the chiral critical temperature $T_{c}=150 \mathrm{MeV}$, which is a typical characteristic of phase transition of second order driven by chiral symmetry restoration [10].

Fig. 2 shows a quite different picture at finite current quark masses $(m=0.0053 \mathrm{GeV}$ corresponds to the physical value of the degenerate $\mathrm{u}$, d-quark current mass in our model [28]). There is no singular behavior any more: the peak of the susceptibility becomes not so sharp and pronounced as in the chiral limit; its height is greatly suppressed and 


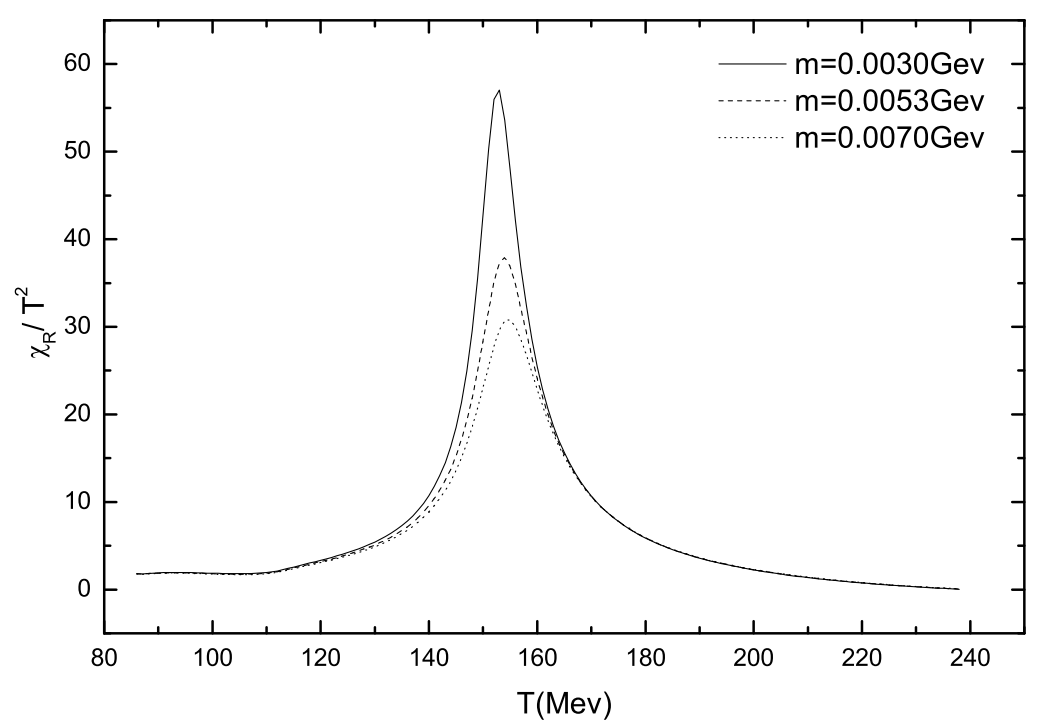

FIG. 2: T-dependence of the renormalized chiral susceptibility at different finite current quark masses. $m=0.0053 \mathrm{GeV}$ corresponds to the physical quark mass in our model.

evidently finite; and there is no unique temperature, but instead a range of finite width where the transition phenomenon takes place. Also notable are the decrease in the height of the susceptibility peak and that the susceptibility peak has a minor shift from $150 \mathrm{MeV}$ to $155 \mathrm{MeV}$ as the current quark mass increases. All of these observations agree well with lattice QCD results [2, 6, 8, 12] and support an analytic crossover involving a rapid change, as opposed to a jump, around the pseudo-critical temperature.

\section{CONCLUSION AND DISCUSSION}

In the present work, we present a continuum investigation of the chiral susceptibility and try to say something about the character of the two-flavor QCD thermal transition from the temperature-dependent behavior of the susceptibility. We first derive a model-independent integral formula, which expresses the chiral susceptibility in terms of basic QFT objects: dressed propagator and vertex. After appropriately regularizing the additive quadratic divergence, we perform a model calculation of the renormalized chiral susceptibility within the DSE-BSE framework with a confining separable model gluon propagator which facilitates summation over Matsubara frequencies. Our model study shows that in the chiral limit, the renormalized chiral susceptibility exhibits a narrow, divergent peak at chiral critical 
temperature $T_{c}=150 \mathrm{MeV}$, signaling a second-order phase transition driven by chiral symmetry restoration. On the other hand, at physical current quark masses, the renormalized chiral susceptibility features a crossover, which involves a rapid, but by no means singular, change in a temperature range of non-vanishing width. So, encouragingly, the lattice QCD observations [2, 6, 8, 12] are reproduced.

Compared to other continuum model studies, like DSE model study [17] and NJL-type model study [15], the present work calculate the chiral susceptibility from an alternative definition (the former two model studies defined the susceptibility as the derivative of the generated mass with respect to the current quark mass and hence the quadratic divergence was circumvented) and in particular from a model-independent expression for the susceptibility. Within the present framework for the chiral susceptibility, the role of the dressed scalar vertex is consistently included into account. However, the present rainbow-ladder DSE-BSE model study still belongs to the class of mean-field approximations, like the two model studies mentioned above. It is interesting to investigate chiral susceptibility beyond mean-field approximations and this problem deserves further study.

\section{ACKNOWLEDGMENT}

We gratefully acknowledge the valuable communication with F. Karsch on the quadratic divergence of chiral susceptibility and its renormalization. Thanks also go to Lei Chang for enlightening discussion on numerical calculations. This work was supported in part by the National Natural Science Foundation of China (under Grant Nos 10575050 and 10775069) and the Research Fund for the Doctoral Program of Higher Education (under Grant No $20060284020)$.

[1] F. Wilczek, Nature 443, 637 (2006).

[2] Y. Aoki et al., Nature 443, 675 (2006).

[3] F. Karsch, arXiv:hep-lat/0711.0656v1; arXiv:hep-lat/0711.0661v1

[4] M. Stephanov, arXiv:hep-ph/0402115v1

[5] D. J. Schwarz, Ann. Phys. 12, 220 (2003); J. Ignatius and D. J. Schwarz, Phys. Rev. Lett. 86, $2216(2001)$ 
[6] Y. Aoki et al., Phys. Lett. B 643, 46 (2006).

[7] J. H. Applegate and C. J. Hogan, Phys. Rev. D 31, 3037 (1985);

[8] M. Cheng et al., Phys. Rev. D 75, 034506 (2007).

[9] Y. Hatta and T. Ikeda, Phys. Rev. D 67, 014028 (2003)

[10] F. Karsch and E. Laermann, Phys. Rev. D 50, 6954 (1994).

[11] C. Bernard et al., Phys. Rev. D 71, 034504 (2005).

[12] M. Cheng et al., Phys. Rev. D 74, 054507 (2006).

[13] Y. Aoki et al., Phys. Lett. B 643, 46 (2006).

[14] L. K. Wu, X. Q. Luo and H. S. Chen, Phys. Rev. D 76, 034505 (2007).

[15] C. Sasaki, B. Friman, and K. Redlich, Phys. Rev. D 75, 074013 (2007).

[16] P. Chakraborty, M. G. Mustafa, and M. H. Thoma, Phys. Rev. D 67, 114004 (2003).

[17] D. Blaschke, A. Holl, C. D. Roberts and S. Schmidt, Phys. Rev. C 58, 1758 (1998); A. Holl, P. Maris, C. D. Roberts, Phys. Rev. C 59, 1751(1999).

[18] J. I. Kapusta and C. Gale, Finite-Temperature Field Theory: Principles and Applications (Cambridge University Press, 2006)

[19] M. He, D. K. He, H. T. Feng, W. M. Sun and H. S. Zong, Phys. Rev. D 76, 076005 (2007).

[20] M. He, Y. Jiang, W. M. Sun and H. S. Zong, Phys. Rev. D 77, 076008 (2008).

[21] Private communication with F. Karsch.

[22] C. D. Roberts, and A. G. Williams, Prog. Part. Nucl. Phys. 33, 477 (1994).

[23] P. Maris and C. D. Roberts, Int. J. Mod. Phys. E12, 297 (2003).

[24] A. Holl, C. D. Roberts, and S. V. Wright, nuch-th/0601071 v1 (2006).

[25] C. D. Roberts and S. M. Schmidt, Prog. Part. Nucl. Phys. 45, S1 (2000).

[26] C. S. Fischer, J. Phys. G32, R253 (2006).

[27] C. J. Burden, L. Qian, C. D. Roberts, P. C. Tandy, and M. J. Thomson, Phys. Rev. C 55, 2649 (1997).

[28] D. Blaschke et al., Int. J. Mod. Phys. A16, 2267 (2001).

[29] J. C. R. Bloch, C. D. Roberts, and S. M. Schmidt, Phys. Rev. C 61, 065207 (2000)

[30] P. Maris, C. D. Roberts, S. M. Schmidt, and P. C. Tandy, Phys. Rev. C 63, 025202 (2001). 